\title{
Alocação dinâmica de largura de banda para redes sem fio infraestruturadas
}

\author{
Julio Cesar T. Guimarães ${ }^{1}$, Henrique D. Moura ${ }^{1}$, \\ Marcos A. M. Vieira ${ }^{1}$, Luiz F. M. Vieira $^{1}$, Daniel F. Macedo ${ }^{1}$ \\ ${ }^{1}$ Departamento de Ciência da Computação \\ Universidade Federal de Minas Gerais (UFMG) \\ \{julio.guimaraes, henriquemoura, mmvieira, lfvieira, damacedo\}@dcc.ufmg.br
}

\begin{abstract}
Communications using wireless networks nowadays receives an increasing amount of users and devices. This implies in an increasing need for improvements in wireless networks. IEEE 802.11ac standard enables channels wider than $20 \mathrm{MHz}$ bandwidth. But currently, access points allocate channel bandwidth statically, independent of the clients' needs. This leads to poor network performance. In this work, we design a system that enables clients to choose the link's bandwidth they connect to the APs. We validate the system through realistic experiments. We show the trade-off between latency and throughput. Our results show that our system improves the frequency spectrum usage, and provide better throughput/latency for the user.
\end{abstract}

Resumo. A demanda por redes sem fio tem crescido nos últimos anos, isto implica em uma maior necessidade de melhoria em seu desempenho. O padrão IEEE 802.11ac permite utilizar canais com largura de banda maiores que 20 $\mathrm{MHz}$. Porém, os pontos de acesso alocam a largura de banda do canal estaticamente, independente das configurações dos clientes. Isso leva a um baixo desempenho da rede. Neste trabalho, propõe-se um sistema que permite aos clientes escolherem a largura de banda para se conectar aos pontos de acesso. $O$ sistema foi validado através de experimentos reais. Destaca-se o compromisso entre latência e vazão. Os resultados mostram que nosso sistema melhora o uso do espectro de frequência.

\section{Introdução}

As comunicações de rede sem fio e móvel estão testemunhando um crescimento exponencial da quantidade de tráfego trocada. As redes sem fio tornaram-se onipresentes e densas. Em um estudo recente ${ }^{1}$, mais de $90 \%$ das universidades dos EUA querem aumentar sua atual infra-estrutura de rede sem fio para suportar a demanda por aplicativos móveis. A mesma tendência é esperada em redes domésticas [Perera et al. 2014] e pequenas redes locais/escritórios domésticos (SOHO) [TechNavio 2017]. Além disso, até 2021, o tráfego de dispositivos sem fio e móveis representará mais de $63 \%$ do tráfego IP total [Cisco 2017]. As redes domésticas tornam-se cada vez maiores devido ao número de dispositivos inteligentes conectados [Perera et al. 2014]. Além disso, novos serviços, como streaming, armazenamento em nuvem e torrents, tornaram-se populares nesses ambientes [Bouchet et al. 2014]. Assim, surge a necessidade de se obter alta vazão nestas redes.

\footnotetext{
${ }^{1}$ http://www.campuscomputing.net
} 
Para melhorar o desempenho dessas redes, são necessárias novas ideias para aumentar a velocidade da conexão, fazendo com que mais dados sejam transmitidos em menos tempo e, de preferência, sem erros.

Para ajudar com essa melhoria, os padrões de protocolo de rede sem fio IEEE 802.11 estão sempre evoluindo. O último padrão que está sendo usado é o 802.11ac [Ong et al. 2011], que usa a frequência $5 \mathrm{GHz}$. Este padrão permite uma velocidade de transmissão maior do que os padrões IEEE anteriores, pois permite, entre outras melhorias, que o espectro de frequência seja dividido em canais de comunicação de diferentes largura de banda. Os canais de comunicação podem ter uma largura de banda variável, com valores entre $20 \mathrm{MHz}$ até $160 \mathrm{M} \mathrm{Hz}$. Esse recurso (largura de banda variável) traz alguns desafios de alocação.

Atualmente, os pontos de acesso (APs) alocam a largura de banda do canal de forma estática, independente das configurações do cliente. Portanto, se um cliente pode se comunicar com uma largura de banda maior do que a largura de banda configurada estaticamente no AP, o cliente não pode usar essa largura de banda. Além disso, se um cliente tiver apenas uma largura de banda pequena e o AP estiver configurado estaticamente com uma largura de banda maior, o cliente não pode se comunicar neste canal com esse AP. Portanto, neste trabalho, tornamos a alocação de largura de banda mais flexível, tornando-a dinâmica, ou seja, a largura de banda em que um cliente se comunica com o AP não precisa ser definida de forma estática a priori. O sistema proposto permite que a largura de banda do canal no AP seja definida por enlace em vez de estaticamente em todo o AP. Assim, cada cliente pode se comunicar com uma largura de banda diferente.

Isso gera novos desafios. Em redes SOHO (Small Offices/Home Offices) no mundo real, é altamente provável ter tipos de dispositivos heterogêneos. Nesse cenário, alguns clientes podem operar apenas em $20 \mathrm{MHz}$ enquanto outros podem operar em $160 \mathrm{MHz}$. Portanto, um cliente não deve degradar o desempenho da rede de outro cliente. Além disso, a desvantagem de canais mais largos é que eles são mais suscetíveis a interferências, uma vez que um canal mais amplo tem uma faixa de espectro de frequiência maior. Além disso, com canais mais largos, o número de canais disponíveis "não sobrepostos" diminui, tornando a interferência mútua um problema. Assim, os canais mais largos devem ser utilizados com sabedoria. Dessa forma, como podemos projetar um sistema para usar a largura de banda do canal eficientemente?

As principais contribuições do deste trabalho são as seguintes: (i) Foi projetado e implementado um sistema que permite aos clientes escolherem a largura de banda do enlace para se conectar aos APs, portanto, a largura de banda entre clientes e APs pode ser configurada dinamicamente. Assim, o AP usa o espectro de frequência de forma mais eficiente, pois, para ambientes com fluxos exigentes, é possível alocar uma largura de banda maior, podendo também usar várias larguras ao mesmo tempo, levando a um aumento considerável na taxa de transferência de dados; (ii) Foi usado uma arquitetura de rede sem fio definida por software para medir métricas na topologia de rede, para clientes sem fio; e (iii) Foi modificado o software dos APs e essas mudanças foram validadas com experimentações físicas.

Este trabalho está organizado da seguinte forma. A Seção 2 apresenta uma discussão sobre as redes sem fio em que se concentra este trabalho, o padrão IEEE 802.11ac, 
e as Redes Definidas por Software (SDN). A Seção 3 apresenta uma comparação deste trabalho com trabalhos relacionados. O sistema proposto é descrito na Seção 4. Na Seção seguinte, os resultados de experimentos físicos são apresentados. Finalmente, na Seção 6, os pensamentos finais e conclusões são apresentados.

\section{Referencial Teórico}

Nesta seção, uma visão geral das redes sem fio e especialmente das redes 802.11ac é apresentada, uma vez que são as redes usadas neste trabalho. O conceito de APs virtuais, que é usado para fornecer várias opções de largura de banda 802.11ac, é explicado. Mostra-se que existe um compromisso entre a vazão e latência para diferentes tipos de aplicações. Esta diferença é explorada neste trabalho para que um cliente selecione a largura de banda mais apropriada para sua aplicação. Finalmente, apresenta-se o conceito de redes definidas por software e a arquitetura utilizada neste trabalho.

\subsection{Redes 802.11ac domésticas e SOHO}

Atualmente, a maioria das redes locais sem fio (WLAN) são redes em um ambiente infra-estruturado [Evans 2011]. Essas redes são compostas por um ou mais APs que são dispositivos responsáveis pela coordenação da transmissão das mensagens sem fio. A comunicação entre os clientes e o AP é direta, em um único salto, utilizando do protocolo IEEE 802.11 (802.11ac, no nosso caso).

Para melhorar a qualidade do serviço prestado pelo Wi-Fi é necessário que o desenvolvimento de padrões de modulação, a codificação e a largura de banda alocada seja melhorado. No contexto das redes baseadas em rádios cognitivos, o acesso dinâmico ao espectro resultou ser uma abordagem promissora para a comunicação nas bandas onde o espectro de rádio já está superlotado. Esta abordagem mostrou um impacto positivo nos níveis de consumo de energia, no tempo de vida da rede e na interferência enfrentada por seus nós [Bukhari et al. 2016].

[Verma et al. 2013] discutem os novos padrões de redes sem fio 802.11ac e 802.11ad. Eles mostram que a vazão de uma rede sem fio pode atingir valores mais altos se os APs alocarem larguras de banda maiores ou se os APs codificarem modulações mais eficientes, ou seja, utilizarem mais pontos na constelação de codificação. A figura 1 mostra a largura de banda e a alocação de canal segundo o padrão IEEE 802.11ac, que usa o espectro $5 \mathrm{GHz}$. O eixo x representa o espectro sem fio e o eixo y representa cada possível alocação de largura de banda. Assim, cada enlace sem fio pode operar em canais de $20 \mathrm{MHz}, 40 \mathrm{MHz}, 80 \mathrm{MHz}$ ou $160 \mathrm{MHz}$. Portanto, quaisquer oito bandas de frequência contíguas de $20 \mathrm{MHz}$ podem ser usadas para atribuir: um enlace para um canal de $160 \mathrm{MHz}$; dois enlaces para canais de $80 \mathrm{MHz}$; quatro enlaces para canais de $40 \mathrm{MHz}$; ou oito enlaces para canais de $20 \mathrm{MHz}$. O intervalo de 5.735 a $5.835 \mathrm{MHz}$ é referido como UNII-3 e não tem canais com $160 \mathrm{MHz}$.

host $A P d^{2}$ é a implementação do AP compatível com o padrão IEEE 802.11 para Linux. Esta implementação possui suporte ao protocolo IEEE 802.11ac, e permite criar vários APs virtuais (VAPs), que são logicamente independentes. Vários VAPs podem ser criados ao mesmo tempo em um único AP físico para fornecer várias redes sem fio

\footnotetext{
${ }^{2}$ Mais informações sobre host $A P d$ podem ser encontradas no site https://w1.fi/hostapd/.
} 


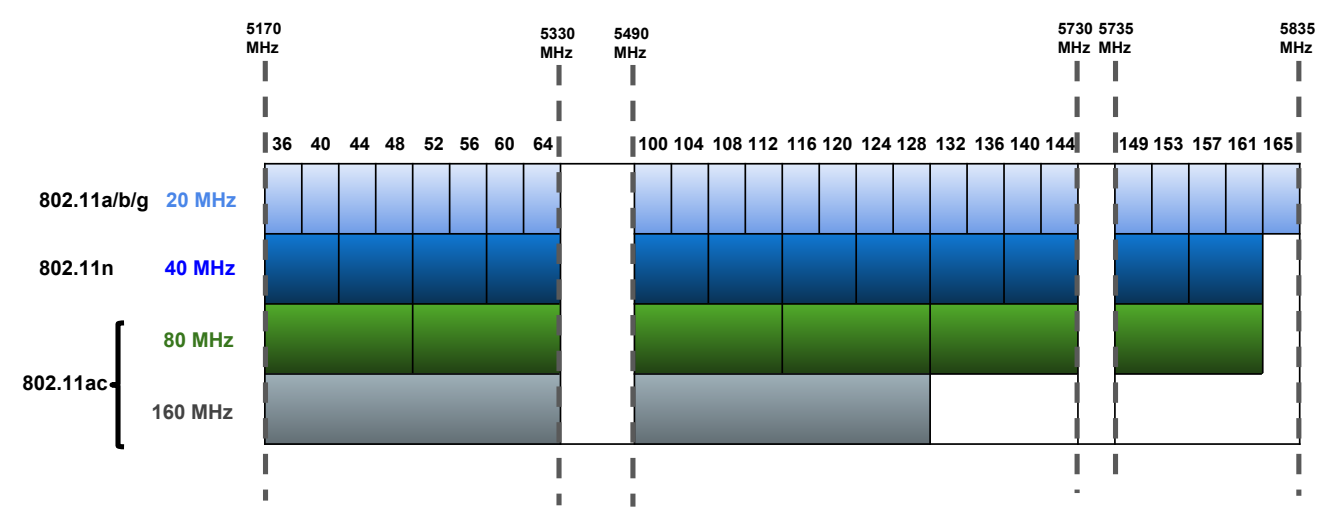

Figura 1. Alocação de canais para IEEE 802.11ac em 5 GHz

virtuais. Cada VAP tem sua própria configuração, portanto, é possível que um único AP físico aceite conexões em diferentes largura de banda, uma para cada VAP criado.

O padrão IEEE 802.11ax introduz suporte a uma modulação 1024-QAM de ordem superior [Gold 2016], em uma camada física nova e redesenhada com o objetivo de melhorar a eficiência espectral global através de OFDMA (Orthogonal Frequency-Division Multiple Access), especialmente em cenários de implantação densa. Este novo padrão deverá fornecer um aumento de até quatro vezes na vazão atual do usuário, mas a previsão da sua submissão para aprovação é apenas em dezembro de 2019, conforme indicado no "Status of Project IEEE 802.11ax"3. A implementação do hostAPd e o hardware utilizados neste trabalho não possuem suporte à este novo padrão, portanto sua avaliação será postergada para trabalhos futuros.

\subsection{Redes Definidas por Software}

As redes definidas por software permitem a criação de uma visão global da rede usando um controlador, tornando mais simples a obtenção de métricas de rede e permitindo controlar a rede com um grão mais fino [Macedo et al. 2015], algo importante para a operação da rede. SDN permite ainda a programação dos elementos de rede [Guedes et al. 2012]. O controlador ainda pode solicitar aos APs informações sobre suas estações.

Uma arquitetura SDN para redes sem fio que fornece controle sobre dispositivos Wi-Fi é o Ethanol [Moura et al. 2015]. O Ethanol atua em redes IEEE 802.11, permite configurar os recursos sem fio e gerenciar redes empresariais e domésticas IEEE 802.11 (WLAN) [Moura et al. 2015]. O Ethanol define uma interface sul que controla os pontos de acesso IEEE 802.11, bem como as estações sem fio que implementam integralmente os padrões IEEE 802.11/2016. Ethanol fornece somente uma plataforma básica SDN e não possui mecanismos de controle implementados, que são uma contribuição de nossa proposta. Desta forma, foram implementados sobre ele um sistema de controle de banda e um agente para o cliente sem fio, capaz de executar o Algoritmo 1 mostrado na seção 4.2 .

$\mathrm{O}$ agente Ethanol é executado no host $A P d$ e responde aos pedidos feitos pelo controlador, ou envia mensagens para o controlador em uma forma de editor-assinante (publisher-subscriber). No AP, o agente é incorporado ao software host APd. Como no

\footnotetext{
${ }^{3} \mathrm{http} / / / \mathrm{www}$. ieee802.org/11/Reports/tgax_update.htm
} 
OpenFlow, conexões seguras (Socket SSL) são usadas para a comunicação do controlador com clientes e APs.

\subsection{Comparação entre vazão e latência}

A Tabela 1 mostra uma comparação entre a vazão e a latência para cada tipo de aplicação. Para transmissões de áudio, por exemplo, é necessário uma baixa latência que possa mitigar as comunicações de voz com atrasos, que podem silenciar ou causar erros na recepção de áudio. Por outro lado, nas comunicação de dados, e-mails e conexões remotas a vazão é a métrica mais importante, desta forma o objetivo é aumentar a velocidade de transmissão de dados, de modo que a comunicação entre fonte e destino seja mais eficaz, melhorando a QoS .

Tabela 1. Aplicação vs. Sensibilidade para cada parâmetro de QoS.

\begin{tabular}{|l|c|c|c|c|}
\hline Tipo de tráfego & Vazão & Perda & Atraso & Jitter \\
\hline E-mail & Baixo & Alto & Alto & Baixo \\
\hline Áudio & Muito Baixo & Médio & Alto & Alto \\
\hline HTTP & Baixo & Alto & Alto & Alto \\
\hline Conexão Remota & Baixo & Alto & Médio & Baixo \\
\hline FTP & Alto & Médio & Baixo & Baixo \\
\hline Vídeo Conferência & Alto & Médio & Alto & Alto \\
\hline
\end{tabular}

Pode-se usar o Ethanol para influenciar os parâmetros QoS, como mostrado na Tabela 1, e assim lidar com as necessidades da aplicação. O controlador Ethanol, ao receber uma conexão dos APs virtuais, começa a gerenciar de cada AP o número de clientes, a taxa de transferência e a latência. O cliente, que se conecta ao ponto de acesso virtual (cada VAP é alocado para cada largura de banda), pode definir a melhor configuração de largura de banda para cada aplicação.

A utilização de uma abordagem SDN facilita lidar com o problema de tratar diferentes padrões tráfegos de aplicações que variam ao longo do tempo, em função do maior poder computacional do controlador, que permite criar mecanismos de decisão que avaliem os padrões de um usuário e assim pode decidir pela conexão ao VAP que atenda a um compromisso geral, isto é, forneça para uma estação a melhor qualidade possível com o menor impacto nos demais usuários e, ao mesmo tempo, evitando o efeito ping-pong.

\section{Trabalhos Relacionados}

OpenRF [Kumar et al. 2013] apresenta uma arquitetura cross layer para gerenciar processamento de sinal MIMO que permite os pontos de acesso no mesmo canal cancelarem a interferência nos clientes uns dos outros. $\mathrm{O}$ foco desta proposta é em técnicas de camada física, e ela não manipula a largura de banda variável.

Cisco usou inicialmente a um procedimento de atribuição de canal dinâmico (Dynamic Channel Assignment - DCA) para selecionar o melhor canal, mas DCA realiza a configuração manual da largura do canal [Cisco 2016]. Mais tarde, a Cisco desenvolveu Dynamic Bandwidth Selection - DBS que permite que aos APs, que operem em larguras de canal mais altas, mudarem para uma largura de banda mais estreita [Cisco 2015]. Contudo este recurso não é executado continuamente. Além disso, a diferença da abordagem com a proposta neste artigo é que, no DBS, não é possível definir a largura de banda do canal por cliente e, se houver um cliente que apenas opera a $20 \mathrm{MHz}$, o AP deverá operar 
nesta frequência para todos os clientes, diminuindo o desempenho da rede para clientes com maiores demandas de banda.

Os padrões IEEE $802.11 \mathrm{a} / \mathrm{b} / \mathrm{g} / \mathrm{n}$ permitem a transmissão de dados com diferentes taxas, dependendo da qualidade do sinal no receptor, permitindo, assim, transmissões de velocidades múltiplas. Contudo os padrões anteriores a IEEE 802.11n só trabalhavam com largura de banda de $20 \mathrm{MHz}$ e mesmo o padrão IEEE 802.11n só permite a configuração manual da largura de banda. [Goussevskaia et al. 2012] forneceram um algoritmo aproximado para escalonar offline os enlaces. Em [Goussevskaia et al. 2013], os autores propõem um algoritmo que também atribui a taxa de dados de um conjunto de enlaces, porém eles não consideraram que a largura de banda fosse variável.

Em [Deek et al. 2011,Deek et al. 2014], o impacto do channel bonding é analisado na rede $802.11 \mathrm{n}$. Os autores identificaram um conjunto de características de rede nas quais os canais mais largos podem ser explorados através de channel bonding. Contudo nosso trabalho avalia redes 802.11ac e fornece meios para controle dessas ligações usando SDN. [Costa et al. 2017] estudaram o problema de alocar offline a largura de banda, o canal, a taxa de dados e o intervalo de tempo de um conjunto de enlaces IEEE 802.11ac. Este trabalho é complementar do nosso, pois eles podem usar o nosso trabalho para configurar dinamicamente as larguras de banda.

Um survey recente [Bukhari et al. 2016] relata muitos esquemas, objetivos e formas de utilização de channel bonding em redes sem fio (especificamente em redes celulares, redes Wi-Fi e redes de sensores sem fio). Diversos trabalhos são apresentados neste survey que buscam combinar um conjunto de canais sem fio contíguos e não sobrepostos, a fim de criar um único canal de maior largura de banda, contudo nenhum trabalho relacionado resolve o problema de alocação de largura de banda baseado na aplicação.

\section{Projeto}

Um AP precisa habilitar a comunicação para todas as larguras de banda permitidas para lidar com os requisitos de aplicações diferentes, conforme mostrado na Seção 2.3. No sistema proposto foram criadas VAPs no AP físico para cada largura de banda disponível. Quando um cliente se conecta a um AP, geralmente ele escolhe o AP que possui a maior força de sinal. No entanto, diferentes aplicações têm necessidades diferentes. O AP físico cria uma fila para os seus VAPs e, geralmente, lida com os pacotes usando uma estratégia FCFS (First Come First Served), de modo que a placa de rede do AP pode funcionar até $n$ VAPs diferentes, cada com uma configuração diferente. O AP possui um buffer para armazenar os pacotes das estações associadas a todos os VAP e os pacotes são encaminhados de acordo com a ordem de chegada.

\subsection{Implementação}

Foi utilizado o Ethanol para controle da rede, e como esta é uma arquitetura aberta, foi desenvolvido um módulo para facilitar o uso do Ethanol no experimento, oferecendo controle automático e criação de pontos de acesso. Esta aplicação é responsável por gerar solicitações de informações, como, por exemplo, saber qual o uso do espectro de cada AP, com o qual é possível gerar relatórios de gerenciamento. Ela permite ainda a criação de VAPs e alocação de largura de banda usando o escalonador padrão do Linux já atribuído às VAPs, que é uma fila simples formada por um buffer. O AP físico processa os quadros 

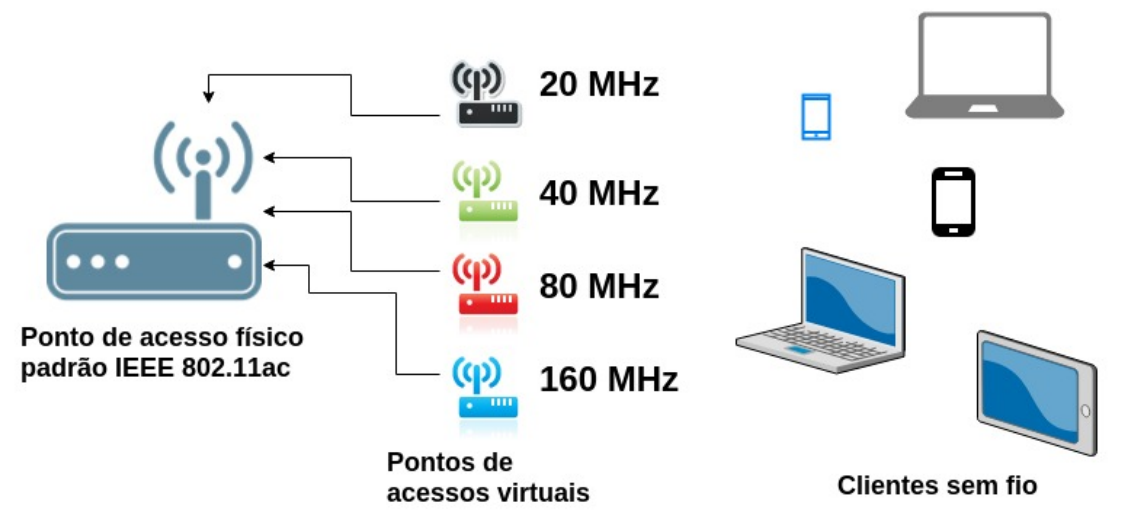

Figura 2. Conexão dos APs virtuais e suas respectivas estações. Cada cor indica uma largura de banda de canal diferente. Um AP físico pode ter muitos VAPs.

de acordo com a sua ordem de chegada para cada VAP. Finalmente, um script foi usado para a geração de tráfego entre as estações e a execução dos experimentos no ambiente real.

\subsection{Seleção de largura de banda}

A estação executa um código simples que seleciona a largura de banda apropriada como mostrado em Algoritmo 1. Na linha 2, o agente carrega um mapeamento que relaciona o tipo de aplicativo (traffic_type $\in \mathbb{N}$ ) para uma prioridade $p \in \mathbb{N}$. Então, em cada iteração, que leva o tempo $t_{\text {intervalo, }}$ o agente executa as instruções no loop. Primeiro ele detecta entre os processo da estação quais tipos de aplicações estão sendo executados, retornando em apps todos type_traf fics encontrados. Na linha 5, o algoritmo seleciona o tipo de tráfego mais importante ( $a p p)$. A largura de banda será ajustada para este tipo de tráfego app. Esta é uma abordagem simplista, uma vez que um aplicativo pode ter comportamentos diferentes, por exemplo, um aplicativo de bate-papo pode funcionar como um aplicativo de voz sobre IP ou uma conferência de voz e vídeo. No entanto, como o foco do trabalho não é a determinação da aplicação executada pelo cliente, essa tarefa foi deixada para o trabalho futuro.

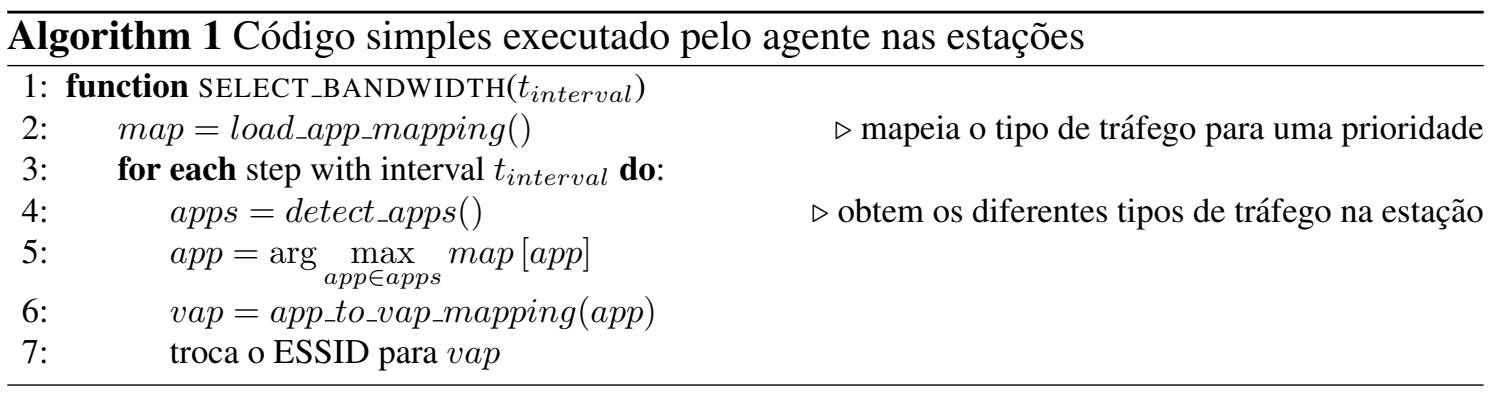

De acordo com um mapeamento predefinido, como o mostrado na Tabela 1, a função app_to_vap_mapping() determina em qual VAP a estação deve se conectar de acordo com o tipo de tráfego. Uma vez que o novo VAP é definido, se o VAP mudou, isto é se o cliente deve migrar para um novo VAP, o agente chama um comando do sistema local para alterar o ESSID (Extended Service Set Identifier), como wpa_cli ${ }^{4}$.

\footnotetext{
${ }^{4}$ https://linux.die.net/man/8/wpa_cli
} 
Se um ponto de acesso virtual tiver mais vazão total do que outros, o controlador SDN pode efetuar o balanceamento de carga entre os clientes. Ethanol pode detectar esse desequilíbrio, por exemplo, se algum VAP tem uma latência global com uma ordem de magnitude diferente dos outros, um evento de handover pode ser desencadeado para transferir alguns clientes no AP desequilibrado para outro AP usando uma mensagem definida pelo padrão IEEE 802.11 denominada Handover Request or BSS Transition Management Request, que será processado pelas estações selecionadas. Um exemplo simples que controla apenas o processo de associação pode ser visto em [Moura et al. 2015]. O controlador também pode interagir com um agente na estação, se a estação não implementar essas mensagens, para que o agente possa executar alguma ação local (por exemplo, para alterar o ESSID). $\mathrm{O}$ algoritmo 1 pode ser alterado para que ele possa receber mensagens de controle do controlador, por exemplo, para alterar os valores do mapeamento na linha 2 ou para mudar para um ESSID definido pelo controlador.

\section{Avaliação Experimental e Resultados}

Nesta seção, uma avaliação experimental do protótipo desenvolvido para a alocação de clientes em diferentes VAPs é apresentado. A seção 5.1 mostra como o experimento foi realizado. Finalmente, a seção 5.2 mostra os resultados das experiências.

\subsection{Avaliação Experimental}

Para validar a solução do problema, os experimentos foram realizados em um ambiente real. A figura 3 mostra o ambiente do experimento realizado. Um computador com Linux Ubuntu 14.04 executa a função do ponto de acesso usando o hostAPd modificado pelo Ethanol. O hostAPd é configurado pelo módulo do Ethanol para criar três redes virtuais. Cada VAP implementa uma largura de banda e SSID diferentes. Configurou-se o controlador SDN Ethanol no mesmo computador. Este controlador poderia estar em outro computador, bastando que o controlador tenha acesso ao ponto de acesso utilizando a rede infraestruturada. O AP que foi utilizado é compatível com todos os demais dispositivos clientes utilizados, uma vez que é capaz de se comunicar usando todas as largura de banda de $20 \mathrm{MHz}$ até $160 \mathrm{MHz}$.

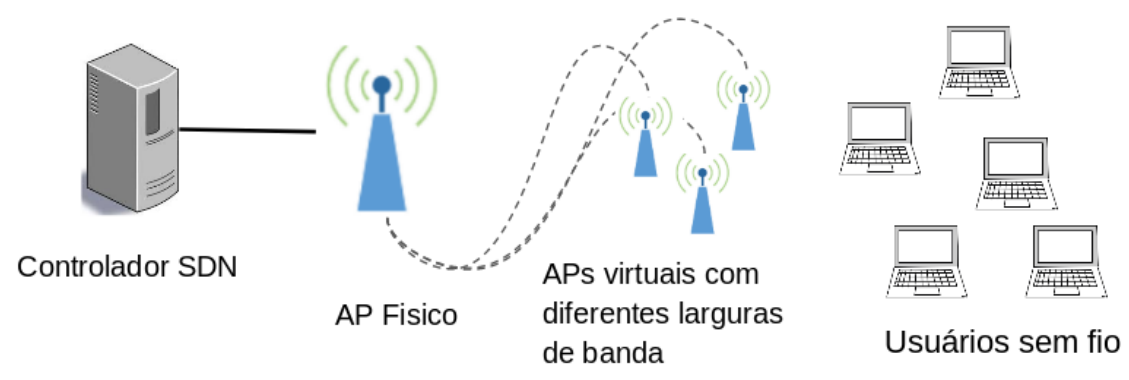

Figura 3. Ambiente experimental

O AP foi construído utilizando um computador do tipo "top-box" baseado em Intel Pentium Quad-core @ 1.9GHz, com 4GB de memória RAM, uma interface Ethernet $1 \mathrm{Gbps}$ (para controle) e uma interface de rede sem fio Intel Wireless 3160 Dual Band AC. As estações utilizaram computadores Intel Pentium com no mínino 2GB, placa Ethernet 1Gbps (para controle) e interface de rede sem fio USB TP-Link Archer T2U V2. Todos 
os computadores rodam Linux Ubuntu versão 14.04. O cenário usou os equipamentos disponíveis para os autores, o que permitiu a criação de um ambiente real de uma homenet. Foram detectados mais de 50 ESSIDs distintos no entorno do ambiente de testes. Portanto as medidas apresentadas neste trabalho apresentam valores sujeitos a interferências não controladas.

A ferramenta iPerf, disponível em https://iperf.fr/, foi utilizada para geração de tráfego e medição de fluxo. Múltiplas estações enviam pacotes ao mesmo tempo para seus respectivos VAPs, desta forma, a fila de pacotes e o escalonador de pacotes do AP são testados. Além disso, utilizou-se ainda o bwping, disponível em https://github.com/ h3dema/bwping-udp, para medir a largura de banda usada e para obter outras métricas de comunicação (por exemplo, atraso) entre dois hospedeiros do mesmo AP virtual e para diferentes APs virtuais.

Neste trabalho, alguns ambientes realistas são configurados para testar o trabalho proposto. Três diferentes configurações de pontos de acesso são testados com largura de banda de 20, 40 e $80 \mathrm{MHz}$. Para os padrões IEEE 802.11n e 802.11ac, a frequência de transmissão está na faixa de $5170 \mathrm{MHz}$ até $5835 \mathrm{MHz}$. Como a banda UNII-3 está sendo usada, o canal da banda começa na frequência $5735 \mathrm{MHz}$. O sistema proposto criou as três redes virtuais com diferentes SSID, usando o centro destes canais e diferentes largura de banda. Todos começam no canal 149, que é o início da banda escolhida (UNII-3). A largura de banda de $160 \mathrm{Mhz}$ não foi utilizada porque as placas de rede no experimento não suportavam essa largura de banda, uma vez que os canais disponíveis para configuração eram apenas referentes a UNII-3, que, como mostrado na Figura 1, tem largura de banda máxima de $80 \mathrm{MHz}$.

Segundo nosso melhor conhecimento, não há outro trabalho relacionado que resolva a alocação de largura de banda para cada fluxo com base na vazão e na latência. Assim, não foi possível comparar outras abordagens, mas a linha de base de $20 \mathrm{MHz}$ é fornecida para comparação com possíveis trabalhos futuros. O presente trabalho experimental usa de cenários simplificados para testar a viabilidade da aplicação e em uma segunda etapa, pretende-se, como trabalho futuro, ampliar o cenário para torná-lo mais complexo.

\subsection{Resultados}

Inicialmente, testou-se três diferentes configurações para os VAPs, com largura de banda de 20,40 e $80 \mathrm{MHz}$, respectivamente. A tabela 2 mostra a média, a mediana e o intervalo de confiança da média para uma confiança de $95 \%$ para as três bandas pesquisadas, sem concorrência entre acessos. Foram realizadas sessenta repetições. Com esses resultados, é possível ver que aumentando a largura de banda, a vazão também aumenta. Esta relação é esperada pela equação de Shannon.

Tabela 2. Vazão média para as três larguras de banda - sem concorrência.

\begin{tabular}{|c|c|c|c|c|}
\hline \multirow{2}{*}{ Largura de banda } & \multirow{2}{*}{$\begin{array}{l}\text { Vazão } \\
\text { Média }\end{array}$} & \multirow{2}{*}{ Mediana } & \multicolumn{2}{|c|}{ Intervalo de confiança $(95 \%)$} \\
\hline & & & Limite Inferior & Limite Superior \\
\hline $20 \mathrm{MHz}$ & 478,7 & 479,4 & 476,8 & 480,5 \\
\hline $40 \mathrm{MHz}$ & 790,8 & 792,4 & 789,5 & 792,0 \\
\hline $80 \mathrm{MHz}$ & 1199,2 & 1198,2 & 1197,1 & 1201,3 \\
\hline
\end{tabular}

Nota: Valores em Mbps. 


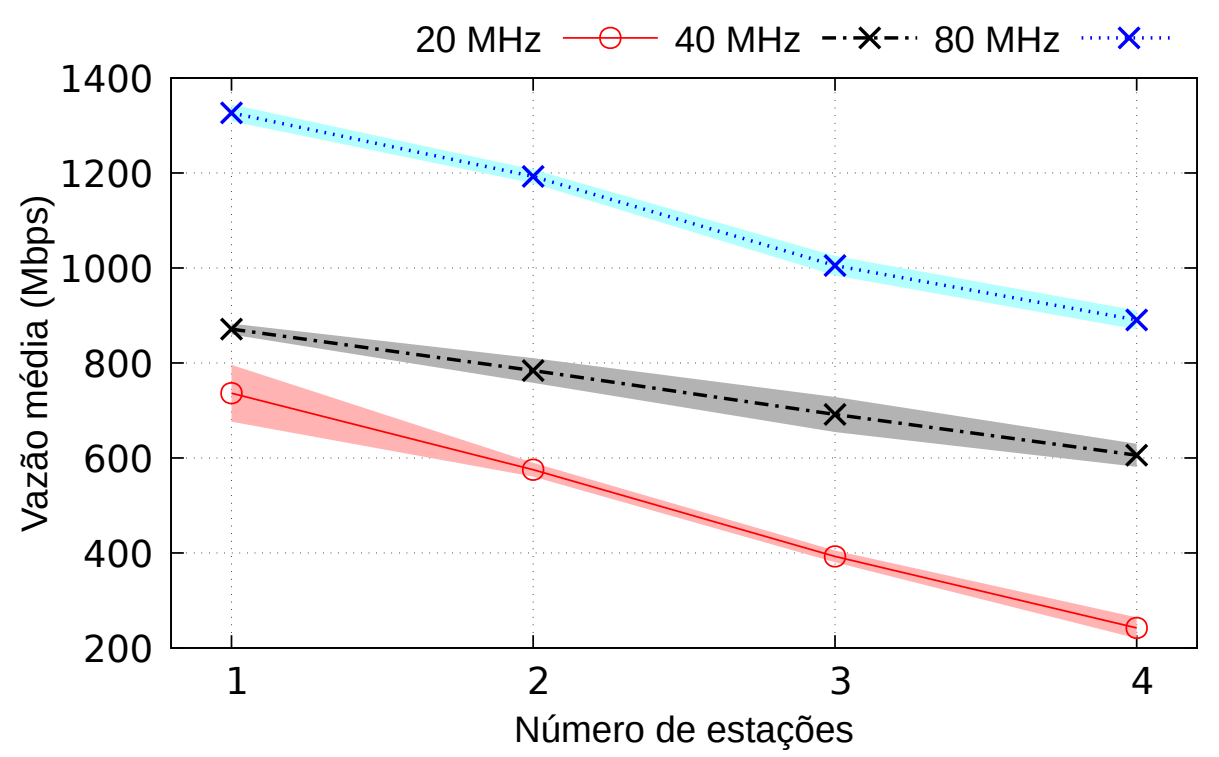

Figura 4. Vazão média versus o número de estações conectadas. Intervalo de confiança de $95 \%$.

Também foi testada a mesma largura de banda com mais estações para ver como o fluxo em cada esquema se comporta. Cada estação tenta ocupar todo o canal disponível. A figura 4 representa a média dos valores obtidos para cada configuração. Em torno de cada curva, uma área correspondente ao intervalo de confiança de $95 \%$ da média foi desenhada. Os resultados também foram esperados, ou seja, um decrescimento da vazão a medida que mais estações são acrescentadas à rede. Infelizmente não possuíamos um grande número de estações e portanto as curvas parecem sugerir um decrescimento linear. Com aquisição de mais dispositivos espera-se poder realizar este experimento em uma escala maior, e verificar quando há saturação da rede.

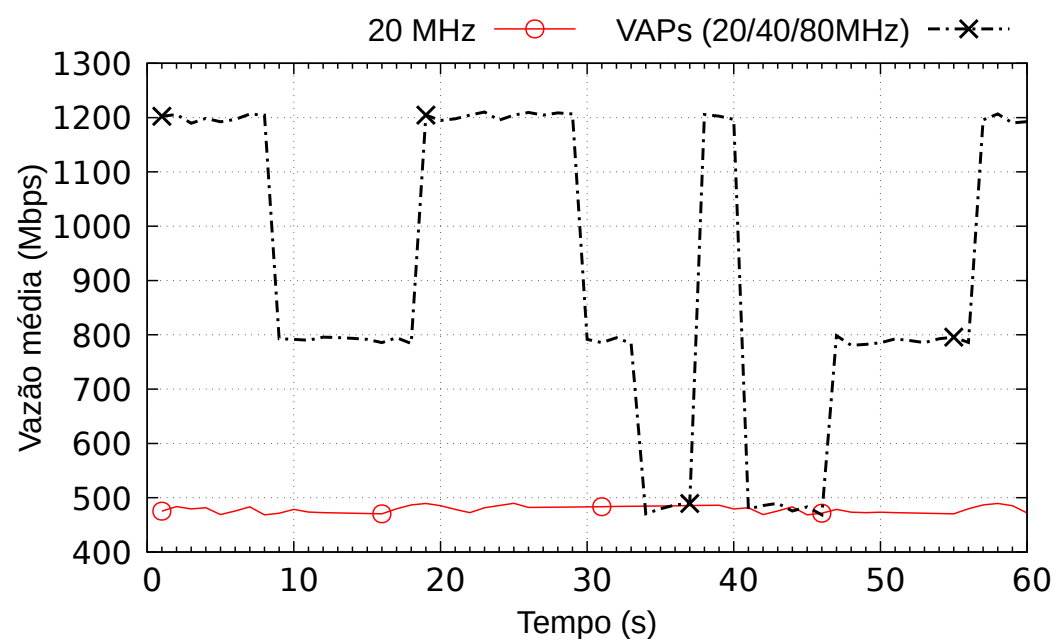

Figura 5. Vazão média por AP para as três larguras de banda vs. valor base com largura estática de $20 \mathrm{MHz}$.

Finalmente, três VAPs foram criados, cada um trabalhando com uma largura de banda diferente. O escalonador das filas de rede dos APs virtuais funciona em base FCFS, assim as menores largura de banda não tem prioridades sobre as demais. Para verificar 
isto, os testes de fluxo foram comparados com uma linha de base que é o AP configurado com largura de banda fixa em $20 \mathrm{MHz}$. A figura 5 mostra os resultados. São mostradas duas curvas. A curva contínua mostra o valor base obtido para uma configuração com somente um VAP de $20 \mathrm{MHz}$. A segunda curva (tracejada) indica a vazão média obtida para uma variação dinâmica da largura de banda em uma estação. Observa-se que a medida que o tamanho da banda aumenta a vazão também aumenta, contudo observa-se que ao dobrar a largura de banda, o mesmo não ocorre com a vazão média na estação. Isto é, a sinalização e o processamento adicionais não permitem que a estação obtenha todo o benefício esperado de uma banda maior.

É necessário analisar o impacto nos requisitos de tráfego ao utilizar IEEE 802.11ac quando há sobreposição de canais, visto que o uso de bandas maiores influencia diretamente a eficiência de transmissão no ambiente. Apesar do problema da sobreposição de canais ter sido tratado por [Jang et al. 2018], este autores não avaliaram o efeito sobre a latência. Este tópico pode ser tratado em uma etapa seguinte do presente trabalho.

\subsection{Vazão vs. Latência}

Para estudo de caso, avaliaram-se os valores de latência e vazão para cada VAP alocado com diferentes largura de banda. $\mathrm{O}$ experimento foi feito usando um único AP físico, com três VAPs, cada um usando uma largura de banda diferente. Um cliente é conectado à um VAP por um período de tempo determinado para testar a vazão e a latência usando duas ferramentas: (1) bwping para medir o atraso e jitter e (2) iPerf para calcular a taxa de transferência em Mbps para cada largura de banda. Neste experimento, esse período foi de uma hora. Era esperado que as condições ambientais fossem diferentes (na região onde o AP está instalado existem outros 50 ESSIDs ao alcance), desta forma o valor médio é influenciado por estas redes, e portanto é necessário executar mais de uma repetição para cada configuração. Os clientes geram fluxos de dados concorrentes para os VAPs. Cada cliente envia pacotes para um VAP.

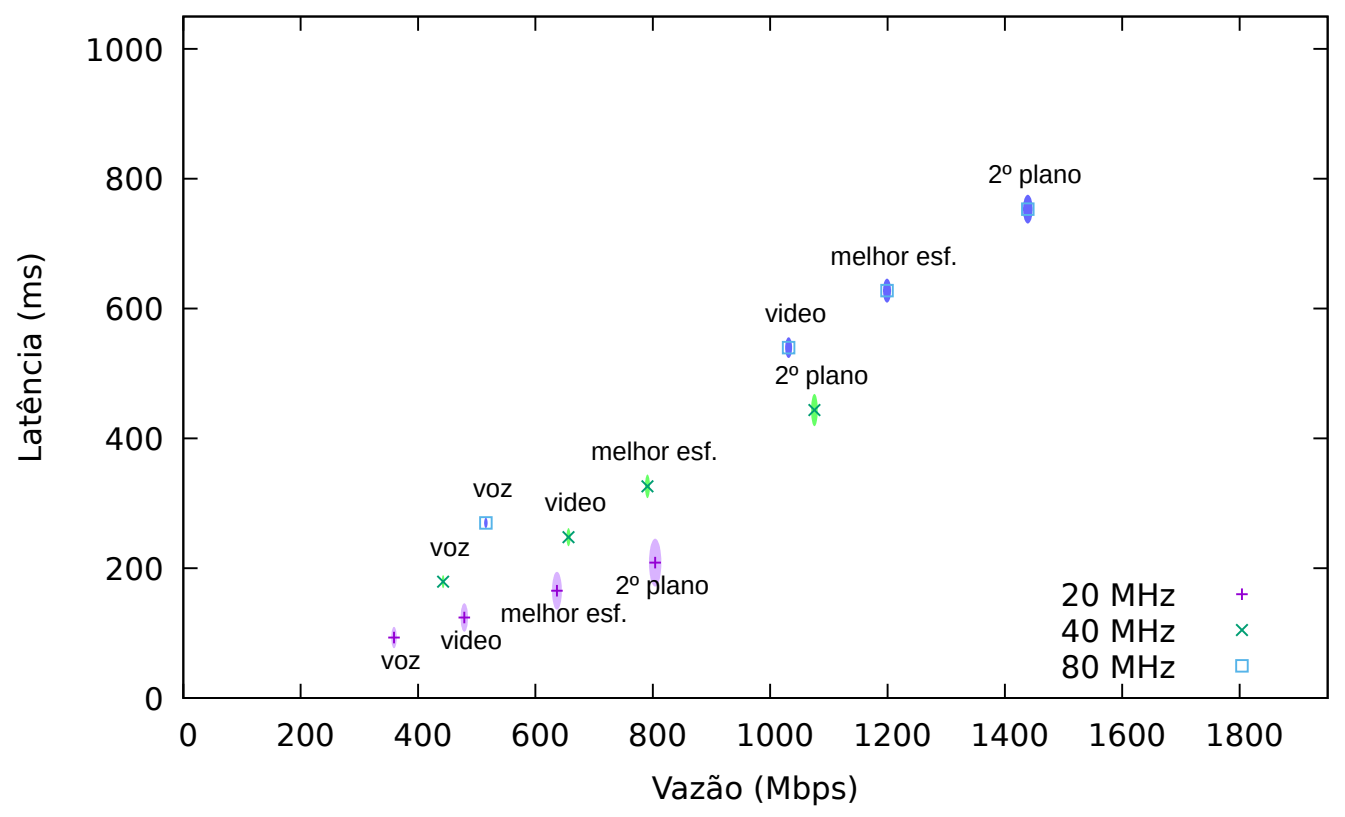

Figura 6. Vazão e latência por largura de banda

A figura 6 mostra um gráfico de dispersão da vazão e latência por largura de banda. 
Para cada largura de banda, existem quatro pontos, que indicam os quatro tipos de serviços no WMM, representando um serviço de voz, um serviço de vídeo, um tráfego de melhor esforço e um serviço em segundo plano. Essas classes são identificadas no gráfico por um rótulo próximo a cada ponto. Cada largura de banda é identificada por diferentes figuras geométricas (e cores) como mostrado na legenda à direita do gráfico. O intervalo de confiança de $95 \%$ nos valores médios é representado por uma elipse desenhada em torno de cada ponto. Os eixos $\mathrm{X}$ e $\mathrm{Y}$ da elipse representam respectivamente o intervalo para a vazão e para a latência. Um VAP com maior largura de banda tem melhores valores de fluxo, o que melhora a transferência de dados e diminui o tempo de resposta, proporcionando ao usuário uma comunicação com maior velocidade. VAPs com menor largura de banda têm valores mais baixos de latência, o que melhora o nível de perda de pacotes e o atraso da transmissão, ideal para aplicações que precisam de algo próximo ao tempo real e com baixa demanda de vazão.

Observamos um comportamento não esperado nesta Figura. A latência está sendo influenciada pela largura de banda. Acreditamos que este comportamento está ligado a capacidade da placa de rede sem fio e dos computadores de processarem o tráfego nestas larguras de banda maiores, e assim a latência fim a fim aumenta.

Usuários egoístas podem optar por selecionar sempre as maiores bandas independente de sua necessidade. O agente instalado no cliente pode incorporar recursos de aprendizado por reforço para aprender a melhor configuração [Moura et al. 2018], e configurado para prover benefício a todos os clientes. O uso de SDN permite ainda implementar um sistema de leilão de banda que também lide com usuários egoístas. Uma aplicação SDN, como a proposta neste trabalho, pode forçar que usuários com este perfil sejam desconectados ou não sejam autorizados a conectar em VAPs com maiores larguras de banda, quando o controlador identificar este comportamento. Estas abordagens serão tratadas em trabalhos futuros.

\section{Conclusão e Trabalhos Futuros}

As contribuições principais deste artigo foram projetar e implementar um sistema que permite aos clientes escolherem a largura de banda do enlace para se conectar aos APs. Um agente foi criado para a estação para realizar as ações necessárias, e o Ethanol forneceu os recursos de SDN para controle da rede sem fio 802.11ac.

Uma boa alocação melhora o desempenho da rede, aumentando a taxa de transferência de dados. O sistema proposto permite um uso mais inteligente do espectro de frequência que leva à uma melhor distribuição da largura de banda do canal entre os APs e seus clientes. Um cliente que suporte somente uma largura de banda de $20 \mathrm{MHz}$ não degrada o desempenho de outros clientes. Para redes com múltiplos APs, como nas redes corporativas sem fio, uma arquitetura SDN para gerenciamento de rede sem fio pode usada para coletar o status da rede e gerenciá-lo. Desta forma, um AP torna-se capaz de alocar dinamicamente sua largura de banda, oferecendo acesso a todas as estações e aumentando a taxa de transferência de dados para os clientes que se comunicam com maior largura de banda.

Pretende-se adicionar um novo escalonador dinamicamente para cada VAP. Desejase investigar o comportamento da rede utilizando diferentes filas de prioridade para diferentes larguras de banda e como esta combinação afeta o desempenho geral do sistema 
considerando diversos tipos de aplicação dos clientes. Em um cenário com mais de um ponto de acesso, um escalonador de conexão pode ser necessário, porque cada cliente escolhe em qual AP ele se conectará. Várias estratégias, por exemplo, seleção aleatória, seleção usando round robin, balanceamento de carga (que considera uma ou mais métricas de rede, por exemplo, sinal, ruído, carga de AP, etc.) podem ser aplicadas a esse cenário. Pretende-se ainda ampliar futuramente os recursos de forma a configurar novos cenários mais complexos. Bem como avaliar a solução proposta com aplicações com padrões de tráfego distinto.

\section{Agradecimentos}

Os autores agradecem as agências de pesquisa CNPq e a FAPEMIG pelo seu apoio financeiro. O presente trabalho foi realizado com apoio da Coordenação de Aperfeiçoamento de Pessoal de Nível Superior - Brasil (CAPES) - Código de Financiamento 001. Este trabalho recebeu financiamento do European Union's Horizon 2020 for research, technological development, and demonstration no âmbito do acordo de subvenção no. 688941 (FUTEBOL).

\section{Referências}

Bouchet, Javaudin, Kortebi, Adbellaouy, E., Brzozowski, Katsianis, Mayer, Guan, Lebouc, Fontaine, Cochet, Jaffré, Mengi, Celeda, Aytekin, G., and Kurt (2014). Acemind: The smart integrated home network. In 2014 International Conference on Intelligent Environments (IE).

Bukhari, S. H. R., Rehmani, M. H., and Siraj, S. (2016). A survey of channel bonding for wireless networks and guidelines of channel bonding for futuristic cognitive radio sensor networks. IEEE Communications Surveys and Tutorials, 18(2):924-949.

Cisco (2015). High Density Experience (HDX) Deployment Guide. https: //www.cisco.com/c/en/us/td/docs/wireless/controller/technotes/8-1/HDX-DG/b $\backslash$ hdx $\backslash$ ddg $\backslash$ final $/ \backslash \backslash$ high $\backslash \_$density $\backslash \_$experience $\backslash$ features $\backslash$ in $\backslash \_$release $\backslash \_8 \backslash \_1 . h t m l$. [Online: accessed in October 29th, 2017].

Cisco (2016). Radio Resource Management White Paper. https://www.cisco.com/c/en/us/ td/docs/wireless/controller/technotes/8-3/b $\backslash \_R R M \backslash$ _White $\backslash$ Paper.html. [Online: accessed in October 13th, 2017].

Cisco (2017). Cisco Visual Networking Index: Forecast and Methodology, 2016-2021. http://www.cisco.com/c/en/us/solutions/collateral/service-provider/ visual-networking-index-vni/complete-white-paper-c11-481360.pdf. [Online: accessed in September 13th, 2017].

Costa, J. M., Paniago, P. P., Noronha, T. F., and Vieira, M. A. M. (2017). A biased randomkey genetic algorithm for the multi-period, multi-rate and multi-channels with variable bandwidth scheduling problem. The 12th edition of the Metaheuristics International Conference (MIC 2017), pages 533-542.

Deek, L., Garcia-Villegas, E., Belding, E., Lee, S.-J., and Almeroth, K. (2011). The impact of channel bonding on $802.11 \mathrm{n}$ network management. In Proceedings of the 7 th Conference on emerging Networking EXperiments and Technologies, page 11. ACM. 
Deek, L., Garcia-Villegas, E., Belding, E., Lee, S.-J., and Almeroth, K. (2014). Intelligent channel bonding in $802.11 \mathrm{n}$ wlans. IEEE Transactions on Mobile Computing, 13(6):1242-1255.

Evans, D. (2011). The Internet of Things: How the Next Evolution of the Internet Is Changing Everything. Technical report. [Online: accessed in September 13th, 2017].

Gold, J. (2016). Faq: What you need to know about 802.11ax, the next big wi-fi standard. https://www.networkworld.com/article/3048196/mobile-wireless/ faq-802-11ax-wi-fi.html. [Online: retrieved in 29-Oct-2017].

Goussevskaia, O., Vieira, L. F., and Vieira, M. A. (2013). Data-rate maximization in wireless communication networks. In PIMRC, pages 3244-3248. IEEE.

Goussevskaia, O., Vieira, L. F. M., and Vieira, M. A. M. (2012). Wireless multi-rate scheduling: From physical interference to disk graphs. Annual IEEE Conference on Local Computer Networks, 37:651-658.

Guedes, D., Vieira, L. F. M., Vieira, M. M., Rodrigues, H., and Nunes, R. V. (2012). Redes Definidas por Software: uma abordagem sistêmica para o desenvolvimento de pesquisas em Redes de Computadores. SBRC 2012.

Jang, S., Shin, K. G., and Bahk, S. (2018). Post-CCA and Reinforcement Learning Based Bandwidth Adaptation in 802.11ac Networks. IEEE Transactions on Mobile Computing, 17(2):419-432.

Kumar, S., Cifuentes, D., Gollakota, S., and Katabi, D. (2013). Bringing cross-layer mimo to today's wireless lans. In Proceedings of the ACM SIGCOMM 2013 Conference on SIGCOMM, SIGCOMM '13, pages 387-398, New York, NY, USA. ACM.

Macedo, D. F., Guedes, D., Vieira, L. F. M., Vieira, M. A. M., and Nogueira, M. (2015). Programmable networks: From software-defined radio to software-defined networking. IEEE Communications Surveys and Tutorials.

Moura, H., Bessa, G. V., Vieira, M. A., and Macedo, D. F. (2015). Ethanol: Software Defined Networking for 802.11 Wireless Networks. IFIP/IEEE International Symposium on Integrated Network Management (IM).

Moura, H. D., Macedo, D. F., and Vieira, M. A. M. (2018). Automatic Quality of Experience Management for WLAN Networks using Multi-Armed Bandit. IFIP/IEEE IM, page 8 .

Ong, E. H., Kneckt, J., Alanen, O., Chang, Z., Huovinen, T., and Nihtilä, T. (2011). Ieee 802.11 ac: Enhancements for very high throughput wlans. In PIMRC, pages 849-853.

Perera, C., Liu, C., Jayawardena, S., and Chen, M. (2014). A survey on internet of things from industrial market perspective. IEEE Access, pages 1660-1679.

TechNavio (2017). Global Consumer, SOHO, and SMB NAS Market 2017-2021. https: //www.researchandmarkets.com/research/lbvtfb/global_consumer.

Verma, L., Fakharzadeh, M., and Choi, S. (2013). Wifi on steroids: 802.11ac and 802.11ad. IEEE Wireless Communications, 20(6):30-35. 